\title{
Simultaneous Bilateral Total Knee Arthroplasty Outcomes
}

\section{Analysed for Present Day Practice}

\author{
Nithin $S^{*}$ \\ Consultant Orthopaedic \& Joint Replacement Surgeon RMM Global Hospital \& Trauma \\ centre, Abu Road, Rajasthan, India
}

*Corresponding author: Nithin Sunku, Consultant Orthopaedic \& Joint Replacement Surgeon RMM Global Hospital \& Trauma centre, Abu Road, Rajasthan, India, Tel: 9886389529; Email: drsnithin@gmail.com

Abbreviations: BTKA: Bilateral Total Knee Arthroplasty; RCTs: Randomized Controlled Trials.

\section{Commentary}

Commentary on an article by Michael-Alexander Malahias "Comparing the Safety and Outcome of Simultaneous and Staged Bilateral Total Knee Arthroplasty in Contemporary Practice: A Systematic Review of the Literature"

Bilateral total knee arthroplasty can be done either in a staged way or simultaneously in one go. Both these ways have their own advantages and disadvantages. There has been lot of controversies going on whether we should do simultaneously or staged. Many studies have been done including RCT's and meta-analysis. Recent trend in replacement is good patient education done preoperatively; minimally invasive surgical techniques, early rehabilitation protocols, and multimodal perioperative pain control have been progressively increasing over the last few years.

Bilateral total knee arthroplasty (BTKA) had done simultaneously in a single sitting have advantages of decreased stay, single anesthesia, and reduced time for physiotherapy and recovery time. It also helps if deformity on both sides to be corrected simultaneously and better rehabilitation. But few disadvantages are increased need for blood transfusions and increased physiological stress induced by simultaneous surgery. Also few studies suggested that inferior technical performance was observed toward the end of the procedure in the second TKA. Also simultaneous BTKA is as effective as staged BTKA in the treatment of bilateral knee osteoarthritis in regards to revision-free survivorship.

In this study by Michael-Alexander Malahias, et al. [1] included 19 good RCTs, total 191,094 patients were included in this review. Sixty three thousand patients underwent simultaneous BTKA, while 105,415 patients underwent staged BTKA during different hospitalizations ${ }^{1}$. Nearly all from these 7 studies with comparable initial characteristics documented no significant differences in the overall complication rates between the 2 groups in addition to no difference in mortality rate, cardiac complications, revision rate, thromboembolic events, and functional outcomes. Simultaneous BTKA was better compared in terms to staged BTKA.

Another study done to evaluate the effectiveness and safety of the patients receiving simultaneous bilateral TKA (BTKA). A total of 93 consecutive patients with bilateral knee degenerative osteoarthritis were divided into two groups--one-stage group (bilateral TKA under a single anesthetic, $n=54$ ) and two-stage group (bilateral TKA performed during two separate admissions, $n=39$ ). The patients in the both group have a similar incidence of postoperative complications $(p=0.544)$. The one-stage BTKA can significantly $(\mathrm{p}<0.05)$ decrease the operative time, length of stay, and hospital charges, when compared to two-stage BTKA. The patients in one-stage group have 


\section{Journal of Orthopedics \& Bone Disorders}

a significantly $(\mathrm{p}<0.05)$ higher postoperative drainage, amount of hidden blood loss, amount of blood loss, allogeneic transfusion and rate of blood transfusion, compared with those in two-stage group. Patients in both groups had a similar KSS ( $p=0.839)$ and ROM $(p=0.383)$. The findings suggests that one-stage BTKA may be safely performed with similar knee function to those of twostage procedure, and has the added benefit of single anesthetic, reduced costs and decreased hospital stay when compared to two-stage BTKA [2].

Simultaneous bilateral total knee arthroplasty (BTKA) is often performed to treat patients with disabling arthritis of both knees. Another retrospective study, they compared 371 patients who had simultaneous BTKA with 67 patients who had staged ( $<6$ months apart) BTKA. Primary outcomes were perioperative complications, length of stay, and transfusion requirements. Complications included respiratory arrest, pulmonary embolism, and deep surgical-site infection. Overall, there was no significant difference in complication rates between the 2 cohorts. Mean transfusion rate was higher for the simultaneous BTKA group [3].

As with all meta-analyses, the results are only as solid as the quality of the studies included. Given the seemingly robust randomized controlled trials (RCTs) considered, the conclusions presented appear well-founded. This raises the interesting point of the hierarchy of evidence in the surgical and scientific literature.

In a high-volume subspecialty setting in which patients undergoing same-day bilateral TKAs were generally much healthier and younger, we found that same-day bilateral TKAs appeared to be safe [4].

\section{Conclusion}

In conclusion, this valuable article contributes further to the knowledge pool with regard to simultaneous bilateral primary total knee arthroplasty, adding a scientifically sound international perspective. It further reinforces there is moderate evidence to show that simultaneous BTKA is as safe as the staged BTKA.

\section{References}

1. Malahias MA, Gu A, Adriani M, Addona JL, Alexiades MM, et al. (2019) Comparing the Safety and Outcome of Simultaneous and Staged Bilateral Total Knee Arthroplasty in Contemporary Practice: A Systematic Review of the Literature. The Journal of Arthroplasty 34(7): 1531-1537.

2. Zhao YT, Chu HJ, Heng DF, Lei J (2015) Comparison of the effectiveness and safety of one-stage versus twostage bilateral total knee arthroplasty. Acta Orthop Belg 81(4): 784-789.

3. Hadley S, Day M, Schwarzkopf R, Smith A, Slover J, et al. (2017) Is Simultaneous Bilateral Total Knee Arthroplasty (BTKA) as Safe as Staged BTKA?. Am J Orthop (Belle Mead NJ) 46(4): E224-E229.

4. Poultsides L, Memtsoudis S, Gonzalez Della Valle A, De Martino I, Do HT, et al. (2014) Perioperative morbidity and mortality of same-day bilateral TKAs: incidence and risk factors. Clin Orthop Relat Res 472(1): 111-120. 